\title{
The curl of angular velocity and its mechanical significance
}

PU GuangYi ( $\sim$ puguangyi@163.com )

Jiangnan University

Pu Cheng-Xi

Business School of Jiangnan University

Article

Keywords: curl, field theory, vortex ring, fluid mechanics, electromagnetic field

Posted Date: July 2nd, 2021

DOI: https://doi.org/10.21203/rs.3.rs-671861/v1

License: (9) This work is licensed under a Creative Commons Attribution 4.0 International License. Read Full License 


\title{
The curl of angular velocity and its mechanical significance
}

\author{
Pu Guang-Yi ${ }^{1,2 *}$, Pu Cheng-Xi ${ }^{3}$ \\ ${ }^{\text {IS }}$ chool of Mechanical Engineering, Jiangnan University, Wuxi 214122, China \\ ${ }^{2}$ Jiangsu Key Laboratory of Advanced Food Manufacturing Equipment and Technology, Wuxi 214122, China \\ ${ }^{3}$ Business School of Jiangnan University, Wuxi 214122, China
}

\begin{abstract}
The curl of the vector field is widely used in modern field theory, fluid mechanics, mathematics, electromagnetic field, and other fields. In this paper, by introducing an auxiliary vector parameter (We called $\overrightarrow{\boldsymbol{P U}}$ ) whose direction satisfies the right-hand thread rule, the mathematical expression of angular velocity vector curl $(\boldsymbol{\nabla} \times \overrightarrow{\boldsymbol{\omega}})$ was obtained by analogy with the method of defining velocity vector curl $(\boldsymbol{\nabla} \times \overrightarrow{\boldsymbol{v}})$. We also pointed out that the laminar flow of viscous fluid in a circular pipe (Hogen-Poiseuille flow) in nature is a typical real example of angular velocity vector curl $(\boldsymbol{\nabla} \times \overrightarrow{\boldsymbol{\omega}})$. Moreover, a concise mathematical equation (Equation(11)) was given, which could be used to describe some motion characteristics of vortex ring theoretically; Therefore, the motion of a single vortex ring has the dual characteristics of the velocity $\operatorname{curl}(\boldsymbol{\nabla} \times \overrightarrow{\boldsymbol{v}})$ and the angular velocity $\operatorname{curl}(\boldsymbol{\nabla} \times \overrightarrow{\boldsymbol{\omega}})$ at the same time.
\end{abstract}

Key words : curl, field theory, vortex ring, fluid mechanics, electromagnetic field

\section{Introduction}

Gradient, divergence, and curl have been widely used in modern field theory, fluid mechanics, mathematics, electromagnetic field, and other fields. Different from gradient and divergence, the concept of vector field curl is more special, difficult to understand. In terms of physical field, the curl of a vector field is a measure of the field's tendency to circulate about a point-much like the divergence is a measure of the tendency of the field to flow away from a point. Once again we have Maxwell to thank for the terminology; he settled on "curl" after considering several alternatives, including "turn" and "twirl"(which he thought was somewhat racy) ${ }^{[1]}$.

In the advanced mathematics textbooks in many colleges, the concept of the curl is usually understood intuitively from the perspective of mechanics, that is, the curl of velocity's vector is equal to twice the angular velocity (Equation $2)^{[2]}{ }^{[3]}$. However, what is the curl of angular velocity? Are there instances of the curl of angular velocity in nature?

According to Stokes theorem, the angular vector field over a closed path $\mathrm{C}$ is equal to the integral of the normal component of the angular vector field's curl over a surface $S$ for which $C$ is the boundary $\left.{ }^{[2]}{ }^{[4]}\right]^{[5]}$. Therefore, based on the pure's mathematical theory, the curl of angular velocity should exist. However, the curl of angular velocity is seldom reported in the current publicly published literature.

In this paper, by introducing an auxiliary vector parameter (We called $\overrightarrow{\boldsymbol{P U}}$ ) whose direction satisfies the righthand thread rule (Figure 2), the mathematical expression of angular velocity vector curl $(\boldsymbol{\nabla} \times \overrightarrow{\boldsymbol{\omega}})$ was obtained by analogy with the method of defining velocity vector curl $(\boldsymbol{\nabla} \times \overrightarrow{\boldsymbol{v}})$. We also point out that the laminar flow of viscous fluid in a circular pipe (Hogen-Poiseuille flow) in nature is a typical real example of angular velocity vector curl. In addition, the motion of a single vortex ring is an example that includes both the velocity vector $\operatorname{curl}(\boldsymbol{\nabla} \times \overrightarrow{\boldsymbol{v}})$ and the angular velocity vector field $\operatorname{curl}(\boldsymbol{\nabla} \times \overrightarrow{\boldsymbol{\omega}})$.

\section{Mathematical model of velocity vector field curl}

First, we recalled the mechanical model of velocity field $\operatorname{curl}^{[2]}{ }^{[3]}$. The rigid body rotates around the fixed axis $Z$ with an angular velocity of $\overrightarrow{\boldsymbol{\omega}}$, the position vector at a point $M(x, y, z)$ on the rigid body is $\overrightarrow{\boldsymbol{r}}(\overrightarrow{\boldsymbol{r}}=\overrightarrow{\boldsymbol{O M}})$ as shown in Figure 1, the velocity of the point $M(x, y, z)$ is ${ }^{[2]}{ }^{[3]}$ :

$$
\overrightarrow{\boldsymbol{v}}=\overrightarrow{\boldsymbol{\omega}} \times \overrightarrow{\boldsymbol{r}}
$$

Here is the velocity vector in Cartesian coordinates: 


$$
\overrightarrow{\boldsymbol{v}}=\left|\begin{array}{ccc}
\overrightarrow{\boldsymbol{\imath}} & \overrightarrow{\boldsymbol{j}} & \overrightarrow{\boldsymbol{k}} \\
0 & 0 & \omega \\
x & y & z
\end{array}\right|=\left\{\begin{array}{lll}
-\omega y & \omega x & 0
\end{array}\right\}
$$

The curl of velocity vector cross-product may be written as a determinant:

$$
\operatorname{rot} \overrightarrow{\boldsymbol{v}}=\boldsymbol{\nabla} \times \overrightarrow{\boldsymbol{v}}=\left|\begin{array}{ccc}
\vec{\imath} & \vec{\jmath} & \vec{k} \\
\frac{\partial}{\partial x} & \frac{\partial}{\partial y} & \frac{\delta}{\delta z} \\
-\omega y & \omega x & 0
\end{array}\right|=\left\{\begin{array}{lll}
0 & 0 & 2 \omega
\end{array}\right\}=2 \overrightarrow{\boldsymbol{\omega}}
$$

Equation (2) reveals the relationship between the angular velocity field $(\overrightarrow{\boldsymbol{\omega}})$ and the curl of velocity, that is, twice the rotation angular velocity of the rigid disk is the curl of velocity $(\boldsymbol{\nabla} \times \overrightarrow{\boldsymbol{v}})$, which was also the origin of the term "curl" by James Clerk Maxwell.

\section{Mathematical model of the curl of angular velocity}

This paper first introduces an auxiliary variable (we called $\overrightarrow{\boldsymbol{P U}}$ ), whose direction meets the right-hand thread rule, as shown in Figure 2. The displacement vector of point $M(x, y, z)$ in the Cartesian coordinate system is $\overrightarrow{\boldsymbol{r}}$ (that is, $\overrightarrow{\boldsymbol{O M}}$ ), as shown in Figure 3. Analogous to the velocity field $(\overrightarrow{\boldsymbol{v}}$, Equation(1)), the angular velocity of point $M(x, y, z)$ could be defined as ${ }^{[3][4]}$ :

$$
\overrightarrow{\boldsymbol{\omega}}=\overrightarrow{\boldsymbol{P U}} \times \overrightarrow{\boldsymbol{r}}
$$
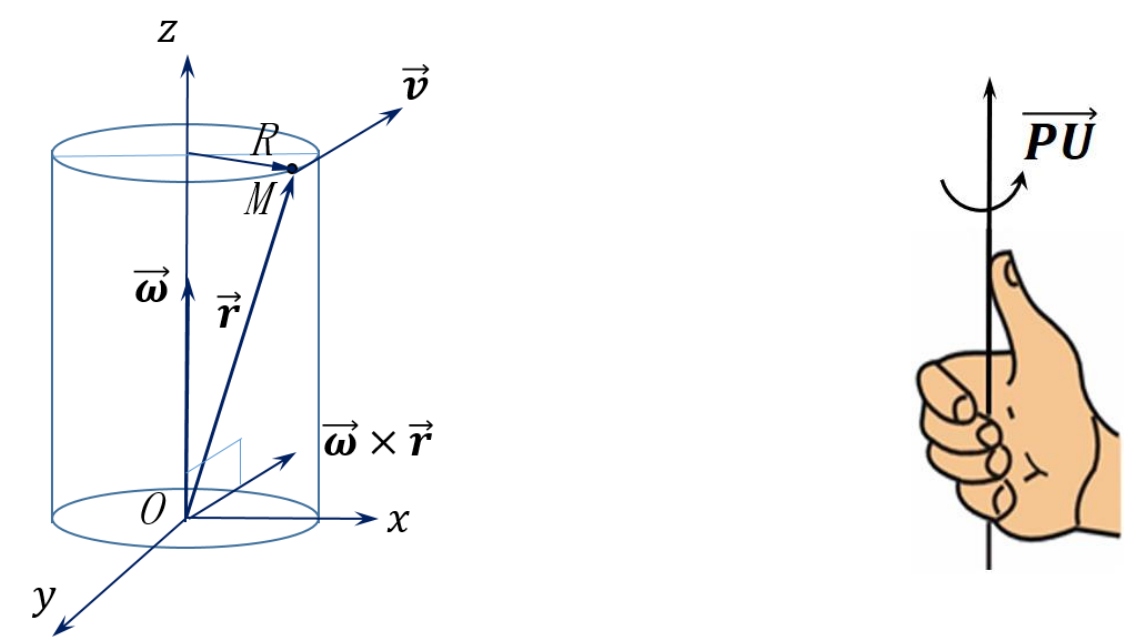

Fig. 1 . The velocity of point $M(x, y, z)$ on a rotating rigid body

Fig. 2. An auxiliary vector parameter $\overrightarrow{\boldsymbol{P U}}$

In Cartesian coordinates, the determinant of angular velocity $(\overrightarrow{\boldsymbol{\omega}})$ could be expressed as:

$$
\begin{gathered}
\overrightarrow{\boldsymbol{\omega}}=\left|\begin{array}{ccc}
\overrightarrow{\boldsymbol{\imath}} & \overrightarrow{\boldsymbol{\jmath}} & \overrightarrow{\boldsymbol{k}} \\
\overrightarrow{P U}_{x} & \overrightarrow{P U}_{y} & \overrightarrow{P U}_{z} \\
x & y & z
\end{array}\right| \\
=\left(z \overrightarrow{P U}_{y}-y \overrightarrow{P U}_{z}\right) \overrightarrow{\boldsymbol{\imath}}+\left(x \overrightarrow{P U}_{z}-z \overrightarrow{P U}_{x}\right) \overrightarrow{\boldsymbol{\jmath}}+\left(y \overrightarrow{P U}_{x}-x \overrightarrow{P U}_{y}\right) \overrightarrow{\boldsymbol{k}}
\end{gathered}
$$

The mathematical definition of the curl of the angular velocity vector field is:

$$
\operatorname{rot} \overrightarrow{\boldsymbol{\omega}}=\boldsymbol{\nabla} \times \overrightarrow{\boldsymbol{\omega}}=\lim _{\Delta s \rightarrow \mathbf{0}} \frac{1}{\Delta s} \oint_{C} \overrightarrow{\boldsymbol{\omega}} \cdot d \overrightarrow{\boldsymbol{l}}
$$

In Equation (5), where $\mathrm{C}$ is a path around the point of interest and $\Delta \boldsymbol{s}$ is the surface area enclosed by that path. The curl of angular velocity $(\boldsymbol{\nabla} \times \overrightarrow{\boldsymbol{\omega}})$ can also be expressed as a determinant in Cartesian coordinates: 


$$
\begin{array}{r}
\operatorname{rot} \overrightarrow{\boldsymbol{\omega}}=\boldsymbol{\nabla} \times \overrightarrow{\boldsymbol{\omega}}=\left|\begin{array}{ccc}
\vec{\imath} & \vec{\jmath} & \vec{k} \\
\frac{\partial}{\partial x} & \frac{\partial}{\partial y} & \frac{\delta}{\delta z} \\
-\omega y\left(z \overrightarrow{P U}_{y}-y \overrightarrow{P U}_{z}\right) & \left(x \overrightarrow{P U}_{z}-z \overrightarrow{P U}_{x}\right) & \left(y \overrightarrow{P U}_{x}-x \overrightarrow{P U}_{y}\right)
\end{array}\right| \\
=2\left(\overrightarrow{P U}_{x} \overrightarrow{\boldsymbol{\imath}}+\overrightarrow{P U}_{y} \overrightarrow{\boldsymbol{\jmath}}+\overrightarrow{P U}_{z} \overrightarrow{\boldsymbol{k}}\right)=2 \overrightarrow{\boldsymbol{P U}}
\end{array}
$$

In addition, the famous Stokes theorem is as follows:

$$
\iint_{S}(\nabla \times \overrightarrow{\boldsymbol{A}}) \cdot \mathrm{d} \overrightarrow{\boldsymbol{S}}=\oint_{C} \overrightarrow{\boldsymbol{A}} \cdot d \overrightarrow{\boldsymbol{l}}
$$

In Equation (7), $\vec{A}$ represents any vector field, if $\overrightarrow{\boldsymbol{A}}$ is the angular velocity field $(\vec{A}=\overrightarrow{\boldsymbol{\omega}})$, then:

$$
\iint_{S}(\boldsymbol{\nabla} \times \overrightarrow{\boldsymbol{\omega}}) \cdot \mathrm{d} \overrightarrow{\boldsymbol{S}}=\oint_{C} \overrightarrow{\boldsymbol{\omega}} \cdot d \overrightarrow{\boldsymbol{l}}
$$

According to Stokes theorem (Equation (8)), that the angular vector field $(\overrightarrow{\boldsymbol{\omega}})$ over a closed path $\mathrm{C}$ is equal to the integral of the normal component of the angular vector field's curl $(\boldsymbol{\nabla} \times \overrightarrow{\boldsymbol{\omega}})$ over a surface $\mathrm{S}$ for which $\mathrm{C}$ is the boundary, as shown in Figure 4, Therefore, based on the pure's mathematical theory, the curl of angular velocity $(\boldsymbol{\nabla} \times \overrightarrow{\boldsymbol{\omega}})$ should exist. While in nature, is there an actual mechanical model of angular velocity curl $((\boldsymbol{\nabla} \times \overrightarrow{\boldsymbol{\omega}})$,Equation 8$)$ ?

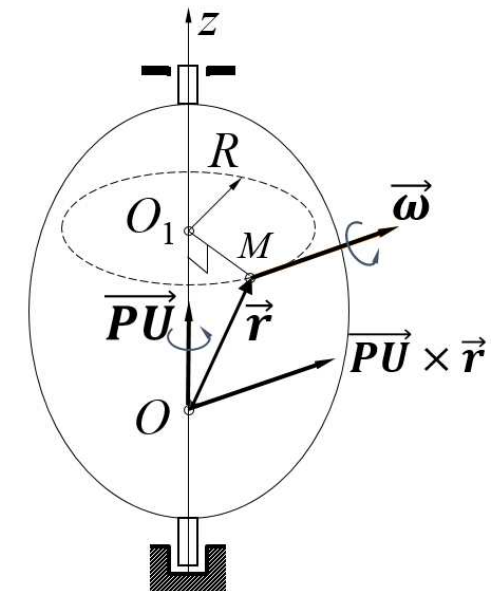

Fig. 3. The angular velocity of point $M(x, y, z)$ on a rotating body

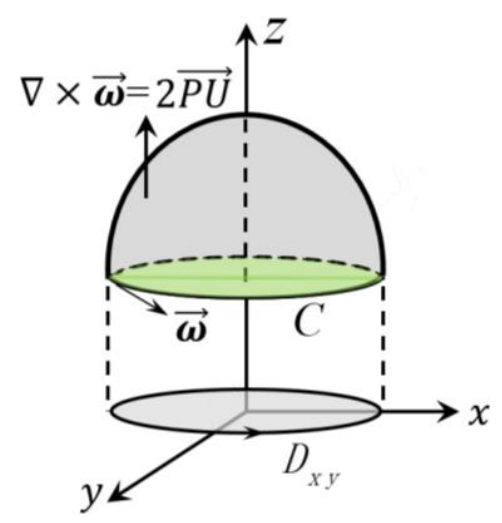

Fig. 4. The Stokes theorem

\section{The real model of angular velocity vector field curl}

Due to the analogy method, equations (1) and (3), (2) and (6) are so similar in form. Equation (2) has a clear mechanical meaning, what about equation (6)?

The first instance(Figure 5): Steady laminar flow of viscous fluid in a circular pipe (Hogen-Poiseuille flow), The fluid velocity vector field distribution is ${ }^{[5][6][7]}$ :

$$
\overrightarrow{\boldsymbol{U}}=\mathrm{U}_{x}=-\frac{1}{4 \mu} \cdot \frac{d p}{d x}\left(R^{2}-r^{2}\right)
$$

Where $\mu$ is the fluid viscosity, $d p / d x$ is the pressure drop along the pipe, $\mathrm{R}$ is the outer diameter of the pipe, and $r$ is the radius at any place of the pipe, as shown in Figure 5. Equation (9) detailed proof process could be found in almost all fluid dynamics textbooks, and the correctness of the Navier-Stokes equations was verified by the HagenPoiseuille flow ${ }^{[5][6]}[7]$.

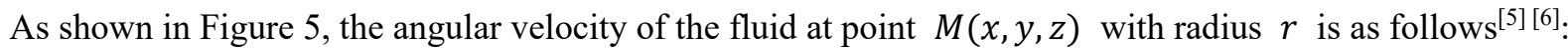

$$
\omega=\frac{\partial U}{\partial r}=\frac{1}{2 \mu} \frac{d p}{d x} \cdot r
$$


Combining equation (3) and equation (6), it was not difficult to derive the auxiliary parameter $\overrightarrow{\boldsymbol{P U}}$ :

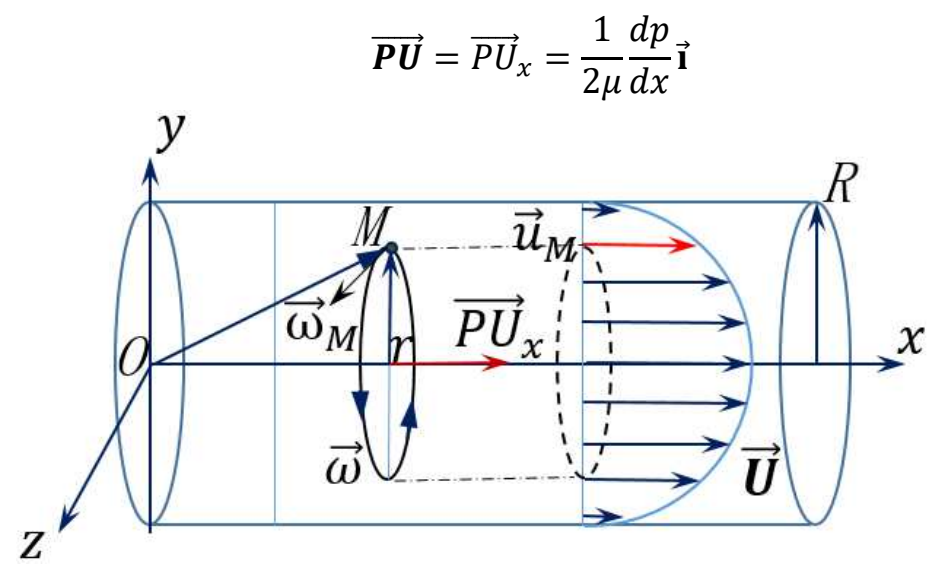

Fig. 5.The velocity distribution of Hagen-Poiseuille fluid flowing in a circular tube

It can be seen from equation (10) that for the Hagen-Poiseuille fluid flowing within a circular tube, there is a horizontal auxiliary parameter $\overrightarrow{\boldsymbol{P U}}_{x}\left(\overrightarrow{\boldsymbol{P U}}_{x}=\frac{1}{2 \mu} \frac{d p}{d x}\right)$ on the cross-section. This case is the intuitive mechanical meaning of the angular velocity $\operatorname{curl}(\boldsymbol{\nabla} \times \overrightarrow{\boldsymbol{\omega}})$.

The motion of vortex rings (such as rings of smoke) in nature is so complicated and fascinating(Figure6), some kinematic characteristics could be described by $\overrightarrow{\boldsymbol{P U}}$ in theory, as the right of equation $(8)\left(\oint_{C} \overrightarrow{\boldsymbol{\omega}} \cdot d \overrightarrow{\boldsymbol{l}}\right)$ is a mathematical description of the circulation of the angular velocity $(\overrightarrow{\boldsymbol{\omega}})$ around a closed path $\mathrm{C}$ :

$$
\Gamma=\oint_{C} \overrightarrow{\boldsymbol{\omega}} \cdot d \overrightarrow{\boldsymbol{l}}=\iint_{S} 2 \overrightarrow{\boldsymbol{P U}} \cdot \mathrm{d} \overrightarrow{\boldsymbol{S}}
$$

Equation(11) could be used to describe some kinematic characteristics of the vortex ring in theory.

From Figure6 and Equation 11, it is not difficult to know, that the motion of a single vortex ring is an example that includes both the curl of the velocity vector $(\boldsymbol{\nabla} \times \overrightarrow{\boldsymbol{v}})$ and the curl of the angular velocity vector field $(\boldsymbol{\nabla} \times \overrightarrow{\boldsymbol{\omega}})$ at the same time.

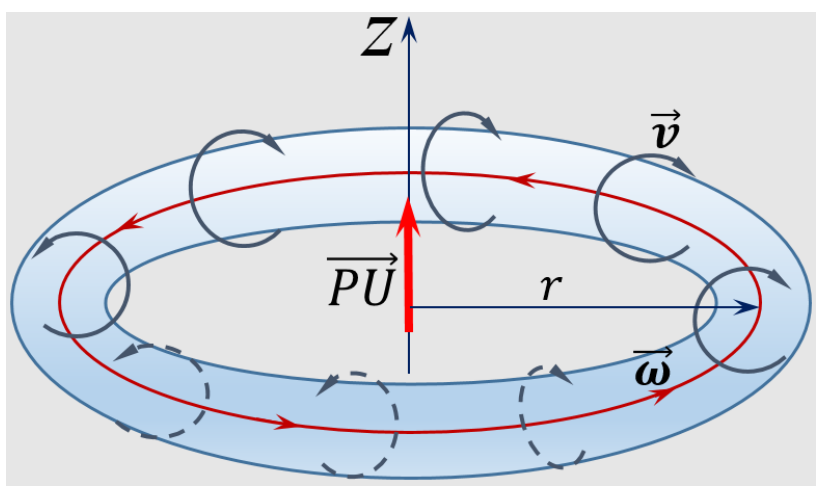

a. The model of a vortex rings

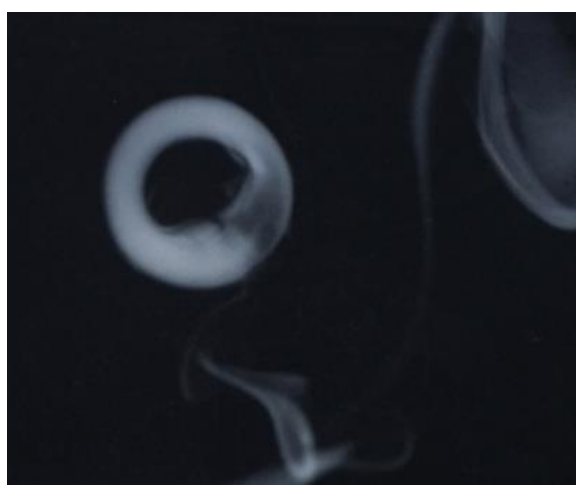

b. rings of smoke

Figure6. Vortex rings\& rings of smoke

\section{Conclusion}

In this paper, by introducing an auxiliary vector parameter $(\overrightarrow{\boldsymbol{P U}})$ whose direction satisfies the right-hand thread rule, the mathematical expression of angular velocity vector curl $(\boldsymbol{\nabla} \times \overrightarrow{\boldsymbol{\omega}})$ was obtained by analogy with the method of defining velocity vector curl $(\boldsymbol{\nabla} \times \overrightarrow{\boldsymbol{v}})$. Based on Stokes theorem and Hogen-Poiseuille flow in fluid mechanics, it 
indicates that the curl of angular velocity $(\boldsymbol{\nabla} \times \overrightarrow{\boldsymbol{\omega}})$ has not only theoretical mathematical significance but also certain mechanical significance.

The curl of velocity $(\boldsymbol{\nabla} \times \overrightarrow{\boldsymbol{v}})$ could be intuitively understood by two times the angular velocity $(\overrightarrow{\boldsymbol{\omega}})$ of the disk in the rigid body mechanics. Similarly, The curl of angular velocity $(\boldsymbol{\nabla} \times \overrightarrow{\boldsymbol{\omega}})$ could be intuitively understood by HogenPoiseuille flow in fluid mechanics (two times of the $\overrightarrow{\boldsymbol{P U}}$ vector).

We hope that the auxiliary $\overrightarrow{\boldsymbol{P U}}$ could be used in more fields, such as for a single vortex ring, $\overrightarrow{\boldsymbol{P U}}$ could also be used to describe its motion intensity in theory.

\section{References :}

[1] Daniel Fleisch, A Student's Guide to Maxwell's Equations. Cambridge University Press, 2006.

[2] Department of mathematics, Tongji University, Advanced mathematics 4th Edition. Beijing: Science Press, 2003.

[3] Department of Theoretical Mechanics, Harbin Institute of Technology. Theoretical mechanics (8th Edition). Beijing: Higher Education Press, 2016.

[4] Richard P. Feynman,RobertB.Leighton, MatthewSands. The Feynman's Lectures on Physics-Volume II(in Chinese). Shanghai: Shanghai Science and Technology Education Press, 2020

[5] Landau, liforsitz, Li zhi. Theoretical physics course: Fluid dynamics 5th Edition (in Chinese). Beijing: Science Press, 2018.

[6] Wang hong wei, Fluid Mechanics as I Understand It. Beijing: National Defense Industry Press,2019

[7] HerbertOertel.Prandtl's essentials of fluid mechanics (in Chinese). Beijing: Science Press, 2019. 